\title{
Hemoptysis during general anesthesia in a diabetic patient with healed tuberculosis
}

\section{-a case report-}

\author{
Mee Young Chung, Hyeon-Do Jeong, Seul-Gi Kim, and Chang Jae Kim \\ Department of Anesthesiology and Pain Medicine, St. Paul's Hospital, College of Medicine, The Catholic University \\ of Korea, Seoul, Korea
}

\begin{abstract}
Hemoptysis is a common complication of pulmonary tuberculosis. Most of the cases of hemoptysis originate from hypertrophied bronchial arteries. Also, diabetes induces pulmonary vascular abnormalities such as endothelial dysfunction, inflammatory infiltration and pulmonary vascular remodeling. A 27 -year-old male, with diabetes and a history of tuberculosis, underwent the procedure of pars plana vitrectomy under general anesthesia. After an uneventful intra-operative period, he had hemoptysis prior to extubation. Emergency fiberoptic bronchscopy showed blood plugs and spotted fresh blood at the right upper lobar bronchus. After successful embolization of the bronchial artery, the patient made a recovery and was discharged without experiencing any complication. Predisposing factors of hemoptysis in this case are presumed to be tuberculosis and diabetes. The bleeding might had been caused by the rupture of a weakened artery within the cavity in the right upper lobe, through expansion of the lung during manual ventilation by positive pressure.
\end{abstract}

Key Words: Diabetes mellitus, Hemoptysis, Tuberculosis, Ventilation.

Hemoptysis is a nonspecific sign associated with serious pulmonary and systemic diseases [1]. The known causes of hemoptysis include infection, cardiovascular diseases, malignancy, and vasculitis [2]. Hemoptysis in a patient with healed tuberculosis during general anesthesia is a rare but often life-threatening complication [1]. We present a case of hemoptysis occurred in a diabetic patient with healed tuberculosis, presumably caused by

Corresponding author: Chang Jae Kim, M.D., Ph.D.

Department of Anesthesiology and Pain Medicine, St. Paul's Hospital, College of Medicine, The Catholic University of Korea, 180, Wangsanro, Dongdaemun-gu, Seoul 02559, Korea

Tel: 82-2-958-2099, Fax: 82-2-967-0235

Email: ksw070591@catholic.ac.kr

Received: July 21, 2016.

Revised: August 24, 2016 (1st); September 7, 2016 (2nd); September 13, 2016 (3rd).

Accepted: September 13, 2016.

Korean J Anesthesiol 2017 February 70(1): 86-89 https://doi.org/10.4097/kjae.2017.70.1.86 rupture of bronchial artery during general anesthesia.

\section{Case Report}

A 27-year-old male (body weight $71.8 \mathrm{~kg}$, height $173 \mathrm{~cm}$ ) with visual disturbance visited the outpatient clinic of ophthalmology. He was diagnosed with vitreous hemorrhage complicated by diabetes. Pars plana vitrectomy under general anesthesia was planned and preoperative evaluation of the patient was reviewed. He had not had any treatment ever since he was first diagnosed with type I diabetes at age 20 , and the treatment began one month prior to the surgery. The patient was also diagnosed with pulmonary tuberculosis when he was 22 -years-old and underwent two years of medication and was claimed completely treated at the age 24 . He had 7 pack-year of smoking history and quit about 9 months prior to the surgery.

Preoperative chest PA showed sequelae of tuberculosis in both lung fields (Fig. 1). Preoperative pulmonary function test (FVC $3.45 \mathrm{~L} \mathrm{FEV}_{1} 2.89 \mathrm{~L}, \mathrm{FVC} / \mathrm{FEV}_{1}$ 83\%) indicated mild re-

(c) This is an open-access article distributed under the terms of the Creative Commons Attribution Non-Commercial License (http://creativecommons.org/ licenses/by-nc/4.0/), which permits unrestricted non-commercial use, distribution, and reproduction in any medium, provided the original work is properly cited. 


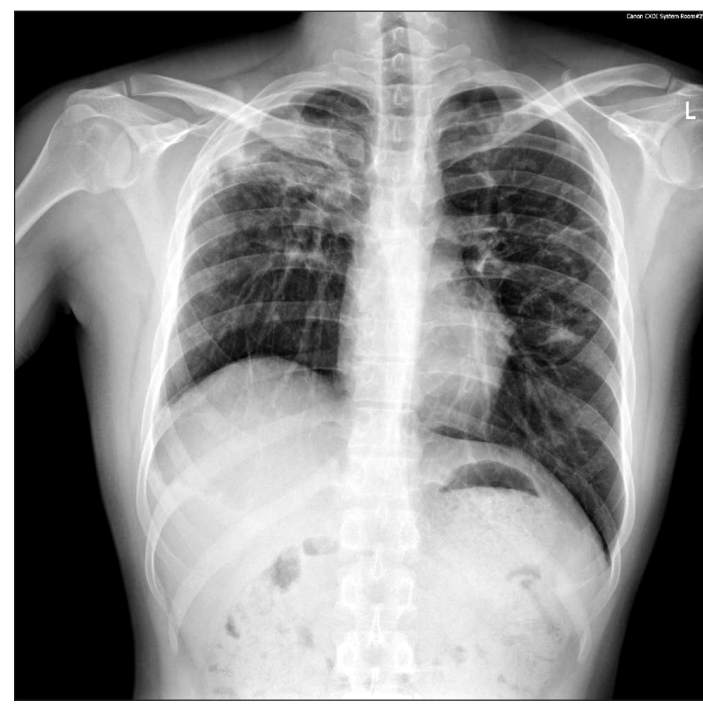

Fig. 1. Preoperative Chest PA showed sequelae of tuberculosis in both lung fields.

strictive lung defect and moderate decrease in diffusing capacity. Preoperative glucose level was $159 \mathrm{mg} / \mathrm{dl}$. He had no signs and symptoms associated with respiratory system. American Society of Anesthesiologists physical status classification was II. The patient was not premedicated. Intraoperative monitorings included noninvasive blood pressure, electrocardiography (lead II, aVL), pulse oximetry, bispectral index, and the end-tidal $\mathrm{CO}_{2}$ $\left(\mathrm{ETCO}_{2}\right)$ concentration measurement. The patient was preoxygenated using mask administration of $100 \% \mathrm{O}_{2}$. Propofol 150 $\mathrm{mg}$ and rocuronium $50 \mathrm{mg}$ were administered intravenously for the induction of anesthesia. Remifentanil was infused continuously using a syringe pump. Laryngoscopy was performed using a Macintosh blade (No. 3) without inducing any trauma. The trachea was intubated using an $8.0 \mathrm{~mm}$ cuffed endotracheal tube. Anesthesia was maintained with $2 \mathrm{~L} / \mathrm{min}_{2}, 2 \mathrm{~L} / \mathrm{min}$ nitrous oxide, and 3-4 vol\% desflurane. The tidal volume was set at $600 \mathrm{ml}$, respiratory rate at 12 per minute and the peak inspiratory pressure was maintained steadily at $16-17 \mathrm{mmHg}$ without extreme deviation throughout the surgery. $\mathrm{ETCO}_{2}$ was maintained at $30-32 \mathrm{mmHg}$. The operation time was 3 hours and 40 minutes in total. The oxygen saturation on the pulse oximetry was $100 \%$ during general anesthesia. Sugamadex $200 \mathrm{mg}$ was administered intravenously for the reversal of muscle relaxation at the end of the operation. Washing out of volatile anesthetics and lung recruit maneuver was conducted simultaneously while assisting patient's respiration through manual ventilation. Then, fresh blood was noted refluxing from the endotracheal tube and tracheobronchial suction was gently performed with $14 \mathrm{Fr}$. catheter. About $200 \mathrm{ml}$ of blood was suctioned in the collecting bottle. The patient's hemodynamic was stable (blood pressure

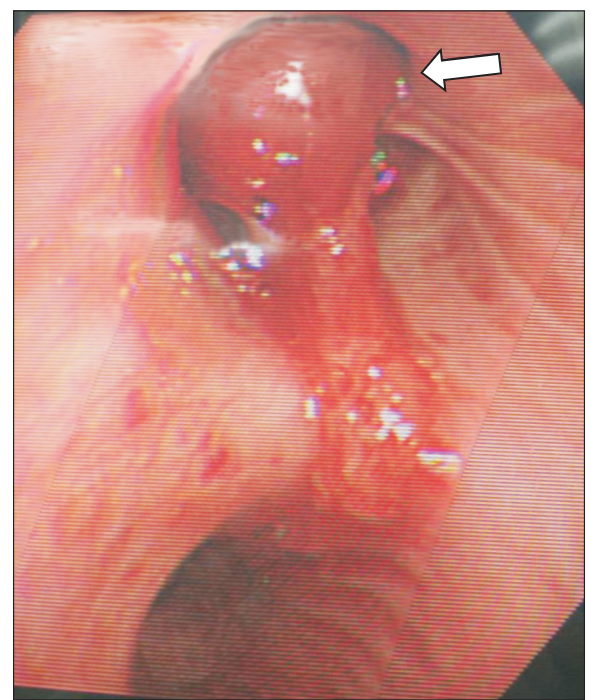

Fig. 2. The blood clot and fresh blood (arrow) was spotted at the right upper lobe bronchus on fiberoptic bronschoscopy.

$110-90 / 60-50 \mathrm{mmHg}$, heart rate $85-95$ beats/min) throughout the incidence and the oxygen saturation on the pulse oximetry was maintained at $100 \%$ with $\mathrm{FiO}_{2}$ 1.0. Based on the concern that the hemoptysis could be aggravated with repetitive coughing, $3 \mathrm{mg}$ of midazolam was administered intravenously for the patient's sedation and to relieve cough, followed by the fiberoptic bronchoscopy in attempt to find the bleeding site. First of all, the presence of airway injury due to endotracheal intubation was evaluated but there was no specific finding in the trachea and main bronchus. No intraoral bleeding was observed. The blood clot and fresh blood was spotted at the right upper lobe bronchus on fiberoptic bronschoscopy (Fig. 2). Because the amount of bleeding decreased gradually during the bronschoscopic examination by the department of pulmonology, the patient was extubated and sent to the postanesthesia care unit, leaving the bleeding site to clot spontaneously. The patient was then transferred to the division of pulmonology and maintained stability with bed rest. Consolidation and cavity with linear scar in right upper lobe appeared on chest CT. Two hundred and fifty $\mathrm{mg}$ of tranexamic acid and $2 \mathrm{~g}$ of ceftriaxone were administered intravenously daily for 5 days, and the blood clots at the anterior and the apical segment of right upper lobe bronchus were shown on the fiberoptic bronschoscopic examination on the 5th postoperative day. However, hemoptysis persisted for 30 days afterwards, which led to the embolization of bronchial artery by the division of interventional radiology on the 36th postoperative day (Fig. 3), after which the symptom was relieved and the patient was discharged. 


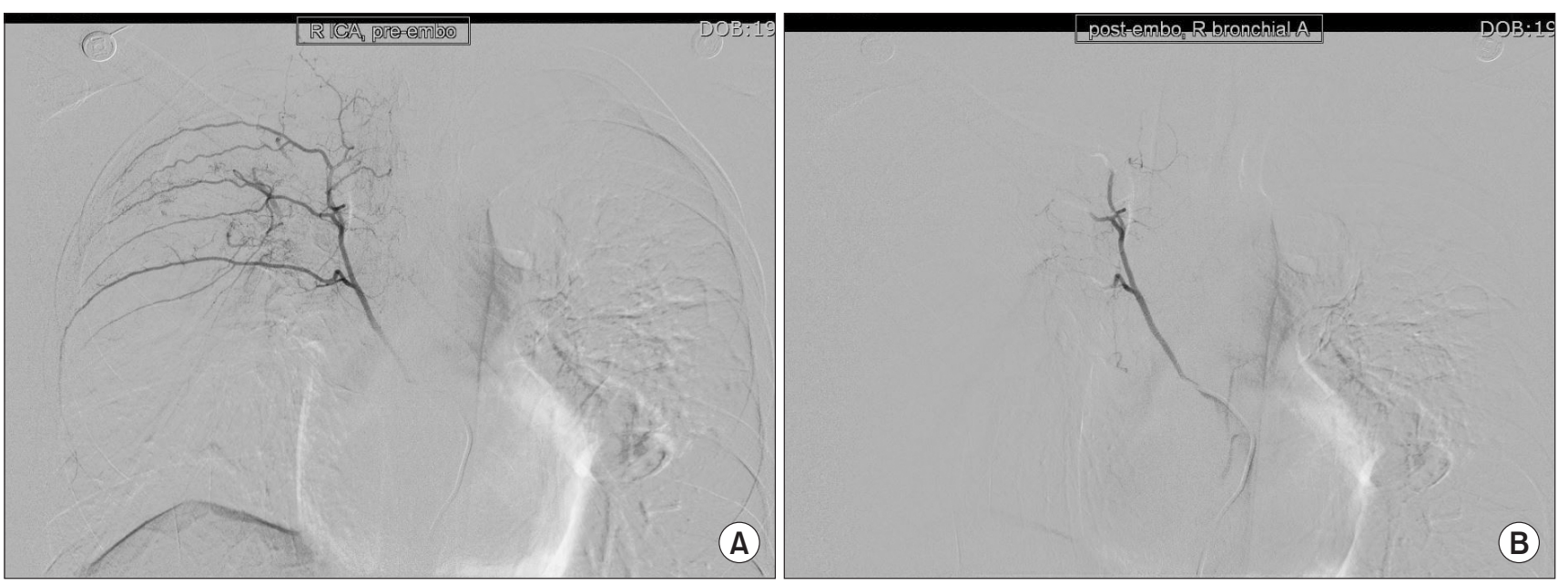

Fig. 3. (A) Angiograhpy before embolization of right bronchial artery. (B) Angiograhpy after embolization of right bronchial artery.

\section{Discussion}

There are many known causes of hemoptysis, including infection, cardiovascular diseases, malignancy, and vasculitis. The most common causes of hemoptysis in the USA are bronchitis (26\%), lung cancer (23\%), pneumonia (10\%), and tuberculosis $(8 \%)$, but tuberculosis is a leading cause of hemoptysis in the developing countries [2]. In addition, the greater frequency of infections in diabetic patients is caused by the hyperglycemic environment that favors immune dysfunction. Especially, patients with diabetes are at higher risk of tuberculosis than those without diabetes [3]. Tuberculosis infection and its treatment may complicate the glycemic control due to the fact that rifampicin may increase the metabolism of the oral antidiabetic drugs [4]. It is suggested that diabetes depresses the immune response (impairing chemotaxis, phagocystosis, and antigen presentation in response to Mycobacterium tuberculosis infection and affecting T-cell function and proliferation) facilitating infection and progression to symptomatic disease $[5,6]$. Also, diabetes results in endothelial dysfunction and lung infiltration that includes the accumulation of macrophages [7]. In this case, the patient had tuberculosis under hyperglycemic state, which was caused by untreated diabetes. In the light of these facts, diabetes might be considered a predisposing factor of hemoptysis. Another common cause of hemoptysis in intubated patient after general anesthesia is a traumatic laryngoscopy and intubation. We excluded this possibility since laryngoscopy was performed using a Macintosh blade (No. 3) without inducing any trauma to the patient, and source of bleeding was confirmed by fiberoptic bronchoscopy [8].

The physical examination may be used to assess the cause, severity, and the bleeding site of hemoptysis. The amount of bleeding is a major determinant of morbidity. Massive hemoptysis (more than 100-600 ml of blood within 24 hours) may cause asphyxiation by aspirated blood which leads to severe hypoxemia and hypotension and is the major cause of immediate mortality [1]. In majority of cases, hemoptysis occur from the hypertrophied bronchial arteries (90\%); a few cases originate from the pulmonary artery which arise secondary to focal weakening of the arterial wall due to inflammatory infiltrates $[9,10]$. Rasmussen described massive hemoptysis in the patient with tuberculosis as bleeding from the erosion of an artery in a thick-walled cavity [1,11]. Wang et al. [1] reported that massive hemoptysis after induction was associated with positive pressure ventilation during general anesthesia. Hemoptysis in this patient was found during manual ventilation at the end of the operation, which suggests that hemoptysis might occur throughout the entire duration of general anesthesia. We speculated that the bleeding might be caused by the rupture of a weakened artery within the cavity in the right upper lobe seen on chest CT, through expansion of the lung during manual ventilation by positive pressure in this case [1].

For the prevention of hemoptysis, preoperative examinations should be carefully reviewed even if the patient with tuberculosis was completely treated. Respiratory symptoms, history taking of physical activity, smoking history, auscultation on chest and chest X-ray should be checked. If necessary, pulmonary function test and chest CT are additionally checked. The minimal limit of airway pressure with adequate minute ventilation sufficient to sustain the $\mathrm{ETCO}_{2}$ within the normal range $(30-35 \mathrm{mmHg}$ ), should be maintained during the entire period of general anesthesia. Especially, recruitment maneuver or manual ventilation should be performed in a controlled and careful manner. Should hemoptysis occur in spite of all these considerations, the treatment depends on the severity and persistence or recurrence of the symptom. It can be controlled by some emergent therapies 
that include medical treatment, bronchial artery embolization and surgical procedure [1]. Usually, the conservative care or medical treatment such as cough suppressant and oral broad spectrum antibiotics is provided for minor hemoptysis [2]. Initial treatment of massive hemoptysis is directed toward airway management, hemodynamic stabilization and early localization of the nature and source of bleeding [11]. Fiberoptic bronchoscope is useful and essential for investigating the cause of hemoptysis as well as for managing the airway [12]. If bleeding has slowed or ceased, cough suppressant to prevent dislodging of hemostatic clots, and supplementary oxygen are used [1]. Patient should be kept in a semi-sitting or recumbent position with the non-bleeding lung positioned slightly superior to the bleeding lung. All expectorated blood and respiratory secretion should be collected to estimate the amount and rate of blood loss. Large bore endotracheal tubes (> $7.5 \mathrm{~mm}$ in diameter) are recommended to facilitate suction. In view of the dual blood supply of the lungs, selective bronchial arteriography should be considered to distinguish bronchial lesions from pulmonary ar- tery lesions such as Rasmussen aneurysm [2]. Bronchial artery embolization is known to be about $90 \%$ effective for the short term control of hemoptysis because bronchial artery lesions are responsible for hemoptysis in approximately $90 \%$ of cases [11]. For the patients with localized lesions and adequate pulmonary function reserve, surgical resection is the most effective therapy [2]. In this case, bronchial arterial embolization was performed owing to persistent hemoptysis in spite of conservative care and medical treatment. Because the embolization effectively stopped the bleeding, further surgical treatment was not considered.

In conclusion, preoperative examinations should be carefully reviewed if diabetic patient had any history of tuberculosis. Even if the patient's tuberculosis was completely treated and currently shows no symptoms associated with respiratory system, any physician or anesthesiologist dealing with such patients must keep in mind the possibility of unexpected hemoptysis that may occur during general anesthesia, and be acquainted with the treatment options if hemoptysis persists.

\section{References}

1. Wang YL, Hong CL, Chung HS, Ho AC, Yu CL, Liu HP, et al. Massive hemoptysis after the initiation of positive pressure ventilation in a patient with pulmonary tuberculosis. Anesthesiology 2000; 92: 1480-2.

2. Johnson JL. Manifestations of hemoptysis. How to manage minor, moderate, and massive bleeding. Postgrad Med 2002; 112: 101-6, 108-9, 113.

3. Restrepo BI, Camerlin AJ, Rahbar MH, Wang W, Restrepo MA, Zarate I, et al. Cross-sectional assessment reveals high diabetes prevalence among newly-diagnosed tuberculosis cases. Bull World Health Organ 2011; 89: 352-9.

4. Dooley KE, Chaisson RE. Tuberculosis and diabetes mellitus: convergence of two epidemics. Lancet Infect Dis 2009; 9: 737-46.

5. Casqueiro J, Casqueiro J, Alves C. Infections in patients with diabetes mellitus: A review of pathogenesis. Indian J Endocrinol Metab 2012; 16 Suppl 1: S27-36.

6. Bacakoğlu F, Başoğlu OK, Cok G, Sayiner A, Ateş M. Pulmonary tuberculosis in patients with diabetes mellitus. Respiration 2001; 68: 595600 .

7. Moral-Sanz J, Lopez-Lopez JG, Menendez C, Moreno E, Barreira B, Morales-Cano D, et al. Different patterns of pulmonary vascular disease induced by type 1 diabetes and moderate hypoxia in rats. Exp Physiol 2012; 97: 676-86.

8. Khanna AK, Cummings KC 3rd. Pulmonary hemorrhage in an outpatient ophthalmic anesthesia setting - it's never "just a cataract". J Anaesthesiol Clin Pharmacol 2012; 28: 520-3.

9. Picard C, Parrot A, Boussaud V, Lavolé A, Saidi F, Mayaud C, et al. Massive hemoptysis due to Rasmussen aneurysm: detection with helicoidal CT angiography and successful steel coil embolization. Intensive Care Med 2003; 29: 1837-9.

10. Corr P. Pulmonary artery aneurysm as a cause of massive hemoptysis: diagnosis and management. Case Rep Radiol 2011; $2011: 141563$.

11. Syed M, Irby J. Airway management of ruptured pulmonary artery "Rasmussen" aneurysm and massive hemoptysis. BMC Res Notes 2015; 8 : 346.

12. Do JY, Kim IS, Hong SJ, Park JH, Shin KM. Hemoptysis after left subclavian central venous catheterization during anesthesia induction for open heart surgery : two cases report. Korean J Anesthesiol 2007; 52: 91-4. 